Supporting Information

\title{
Magnetic Nanowire Biolabels using Ferromagnetic Resonance Identification
}

\author{
Joseph Um ${ }^{*}, \dagger$ Yali Zhang, ${ }^{\dagger}$ Wen Zhou,${ }^{\dagger}$ Mohammad R. Zamani Kouhpanji, ${ }^{\dagger}$ Cosmin Radu,${ }^{\ddagger}$ \\ Rhonda R. Franklin,$^{\dagger}$ and Bethanie J. H. Stadler ${ }^{*, \dagger}$ \\ 'Department of Electrical and Computer Engineering, University of Minnesota, Minneapolis, \\ MN 55455, United States
}

‡ake Shore Cryotronics Inc., Westerville, $\mathrm{OH} 43082$, United States

\section{Corresponding Authors}

*E-mail: umxxx023@umn.edu; stadler@umn.edu 
(a)


(b)
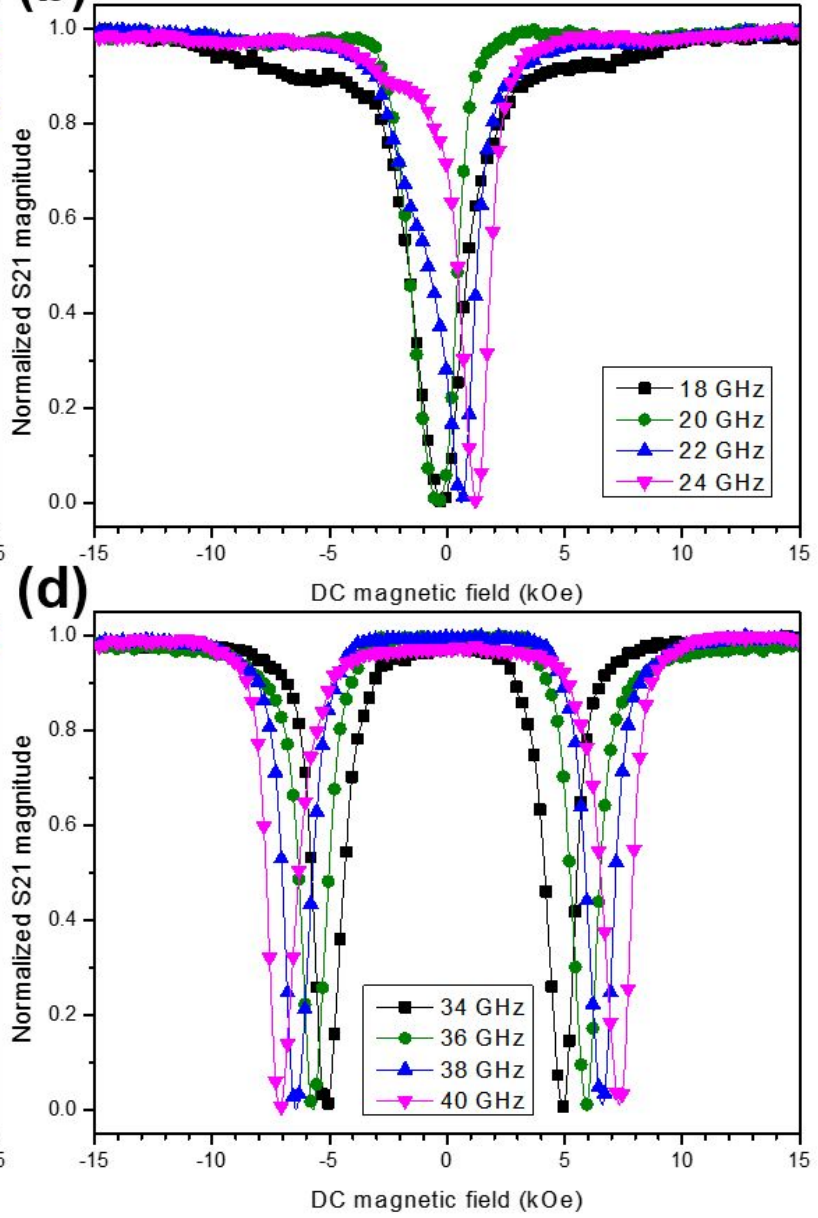

Figure S1. Normalized $\mathrm{S}_{21}$ (microwave signal at the output compared to the input) at (a) 10-16 GHz, (b) 18-24 GHz, (c) 26-32 GHz, and (d) 34-40 GHz for Fe MNWs with DC field parallel to MNW axis. 
(a)



(c)

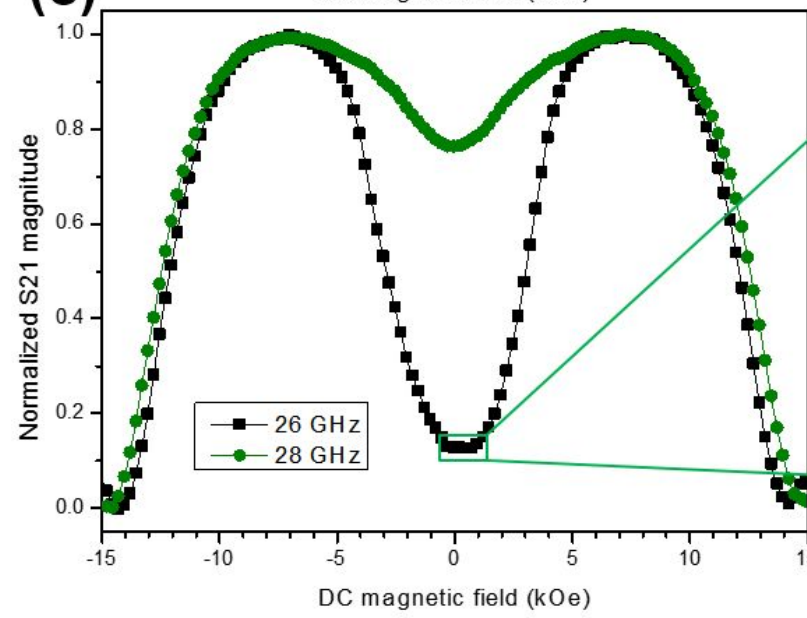

(b)

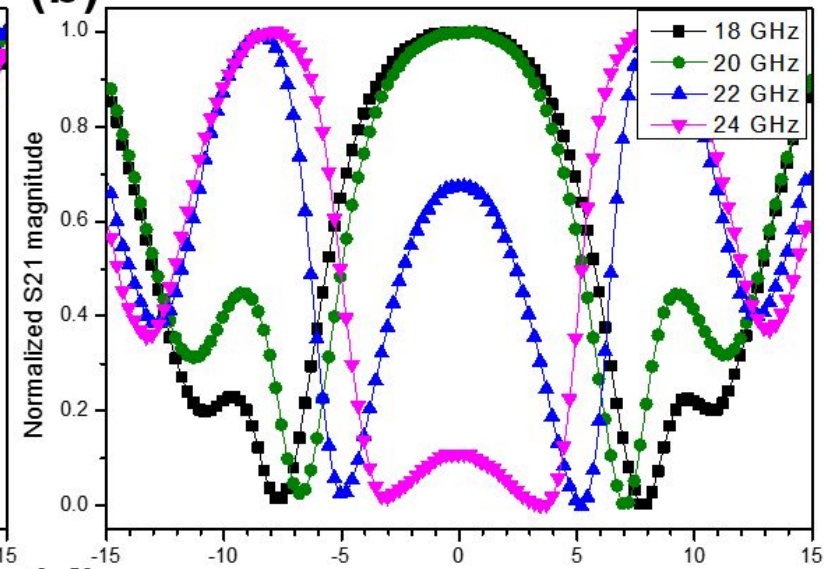

(d)

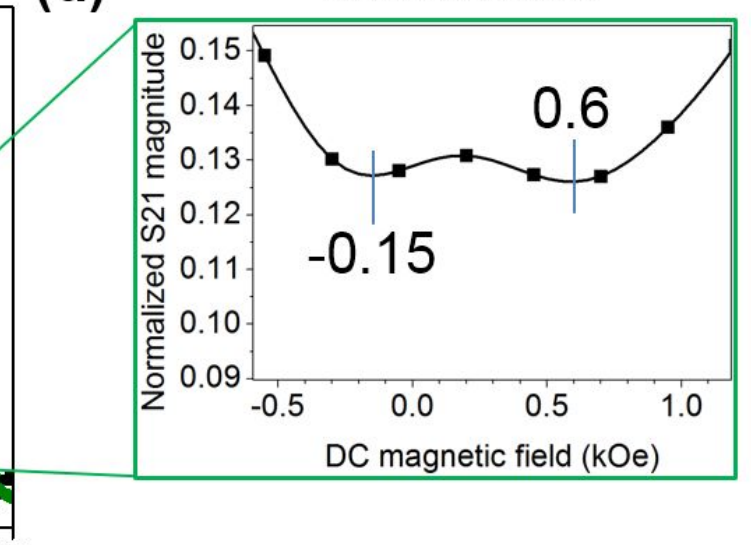

Figure S2. Normalized $S_{21}$ (microwave signal at the output compared to the input) at (a) 10-16 $\mathrm{GHz}$, (b) $18-24 \mathrm{GHz}$, and (c) $26-28 \mathrm{GHz}$ and (d) two FMR absorption nulls of normalized $\mathrm{S}_{21}$ at $26 \mathrm{GHz}$ for $\mathrm{Fe}$ MNWs with $\mathrm{DC}$ field perpendicular to MNW axis. 
(a)
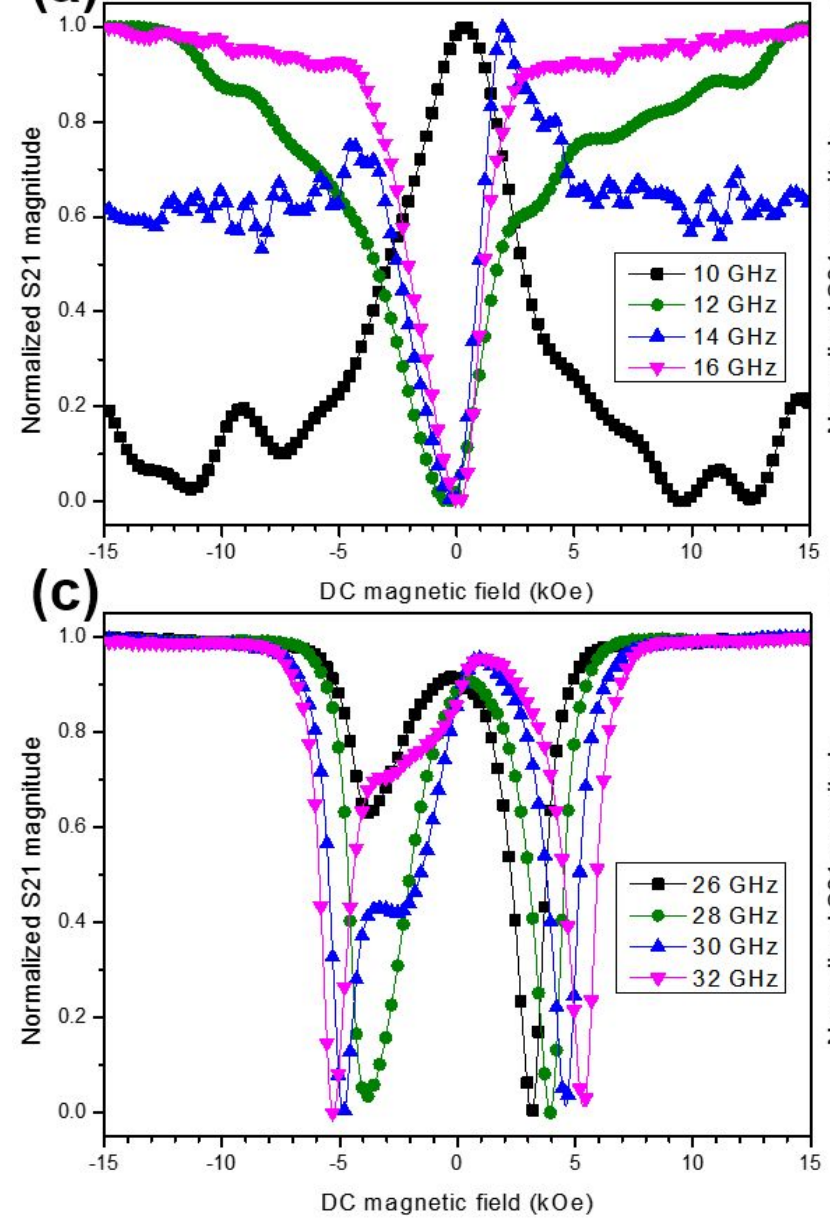

(b)
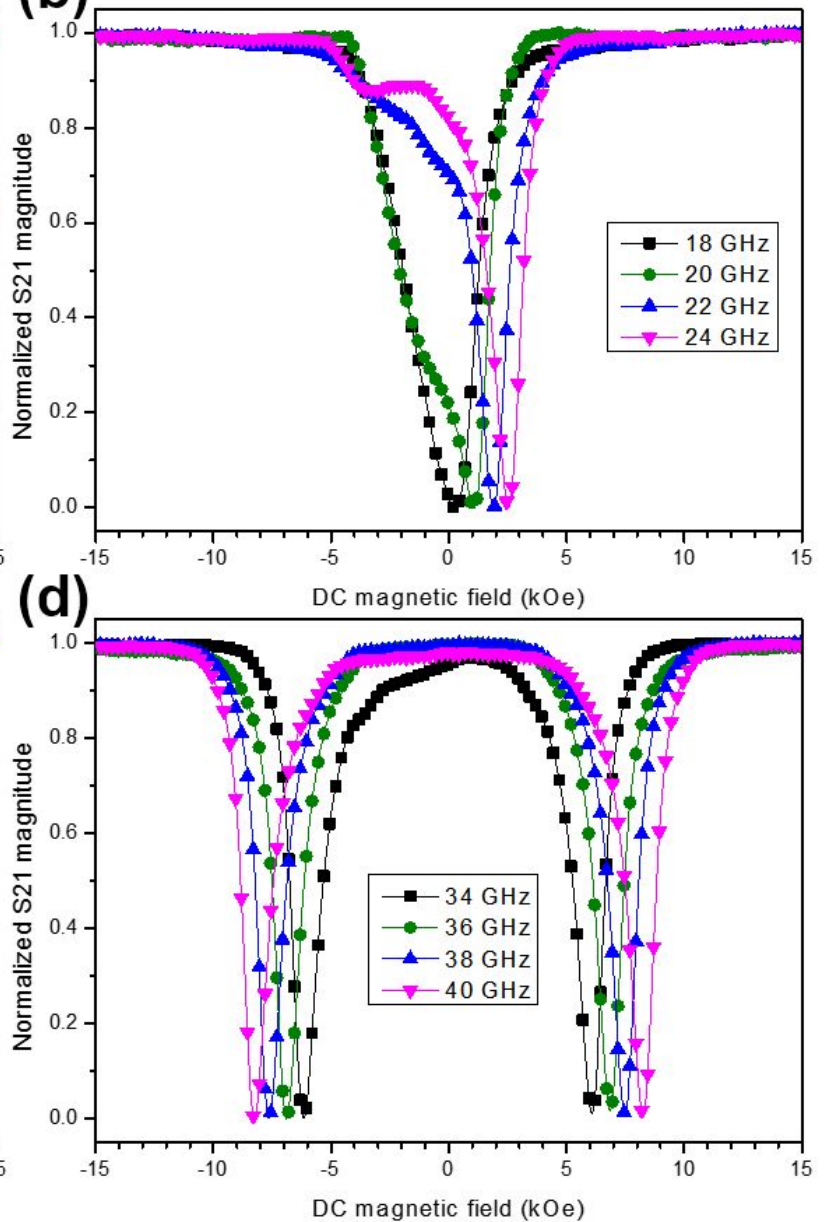

Figure S3. Normalized $\mathrm{S}_{21}$ (microwave signal at the output compared to the input) at (a) 10-16 GHz, (b) 18-24 GHz, (c) 26-32 GHz, and (d) 34-40 GHz for Co MNWs with DC field parallel to MNW axis. 
(a)


(b)

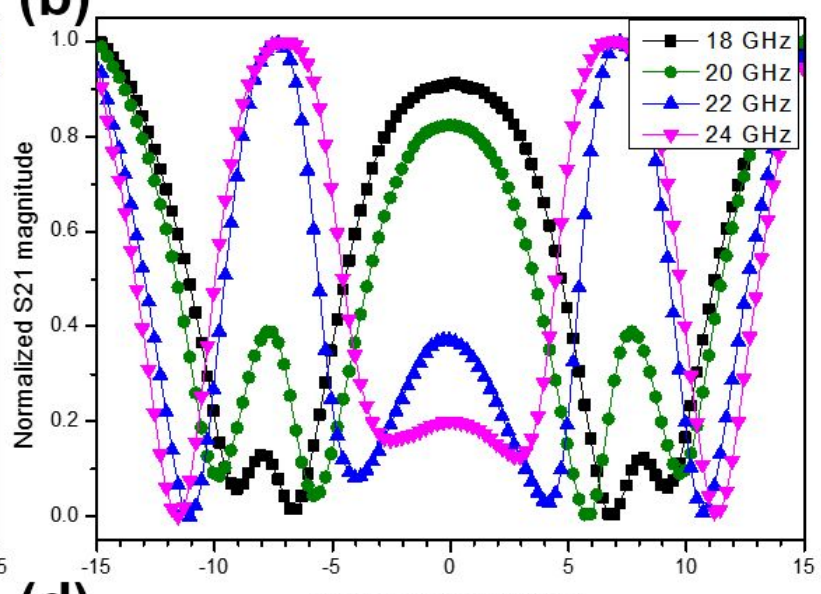

(d)

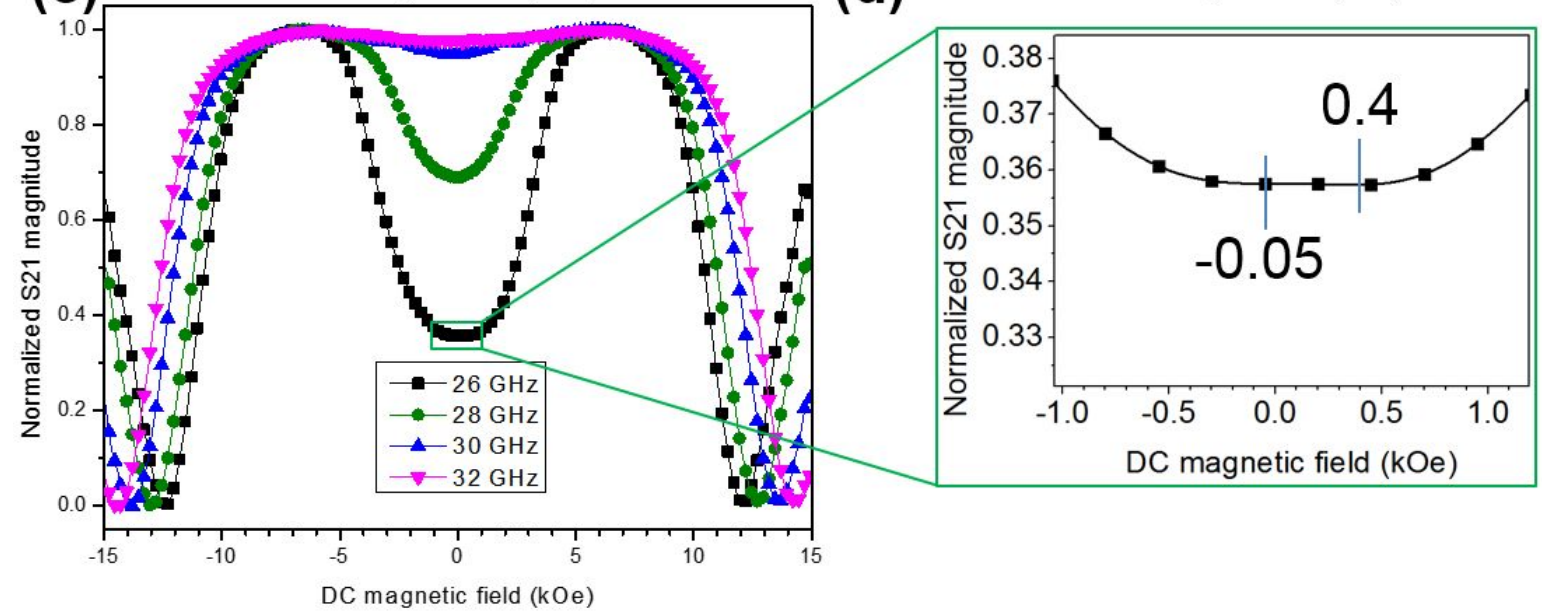

Figure S4. Normalized $S_{21}$ (microwave signal at the output compared to the input) at (a) 10-16 $\mathrm{GHz}$, (b) $18-24 \mathrm{GHz}$, and (c) $26-32 \mathrm{GHz}$ and (d) two FMR absorption nulls of normalized $\mathrm{S}_{21}$ at $26 \mathrm{GHz}$ for Co MNWs with DC field perpendicular to MNW axis. 
(a)
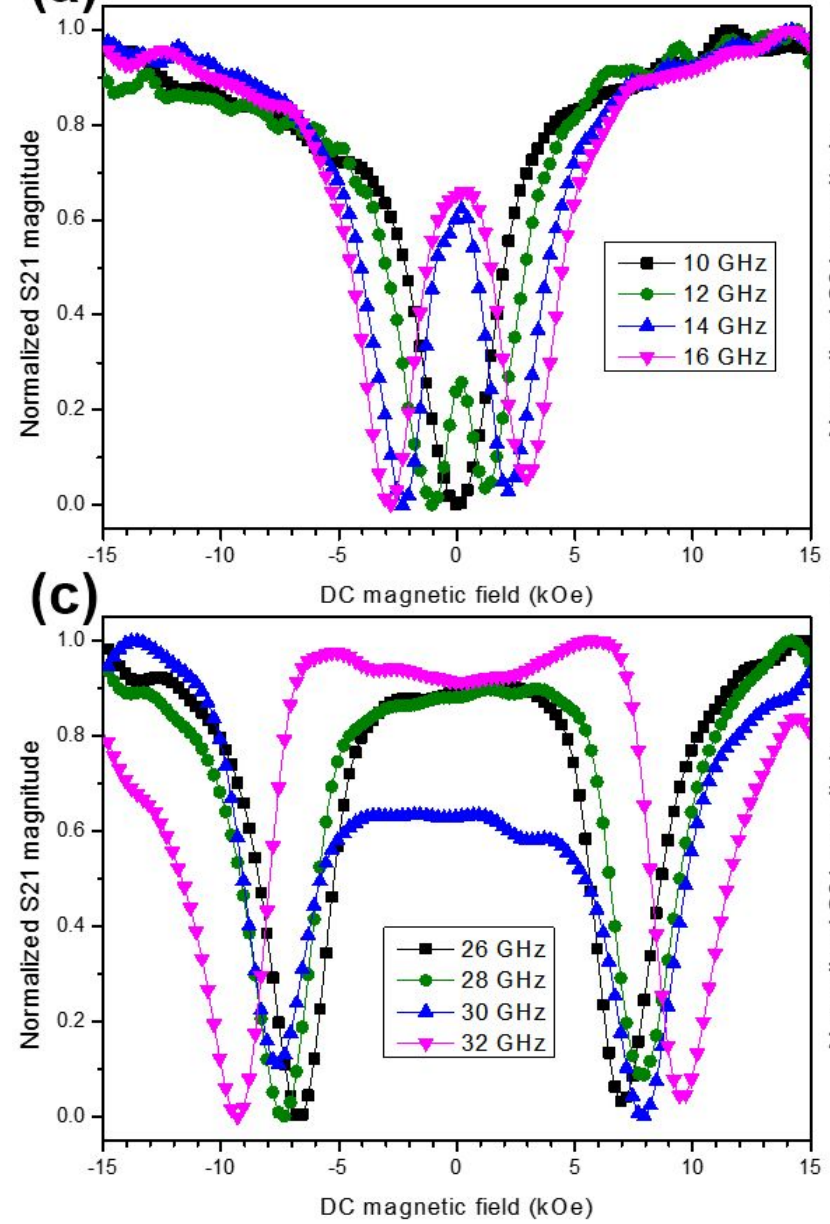

(b)


Figure S5. Normalized $\mathrm{S}_{21}$ (microwave signal at the output compared to the input) at (a) 10-16 GHz, (b) 18-24 GHz, (c) 26-32 GHz, and (d) 34-40 GHz for Ni MNWs with DC field parallel to MNW axis. 
(a)


(b)
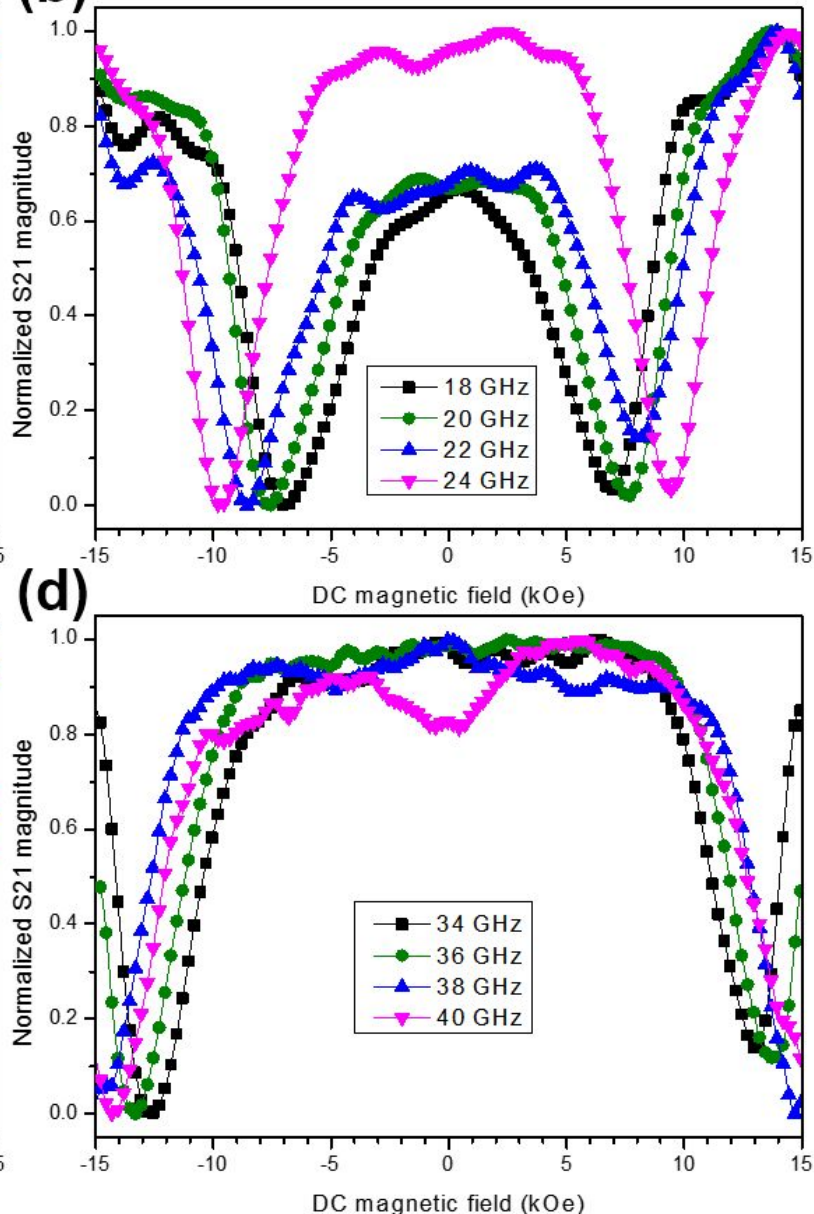

Figure S6. Normalized $\mathrm{S}_{21}$ (microwave signal at the output compared to the input) at (a) 10-16 GHz, (b) 18-24 GHz, (c) 26-32 GHz, and (d) 34-40 GHz for Ni MNWs with DC field perpendicular to MNW axis. 


\section{Error Analysis}

\section{Errors in $\mathbf{S}_{21}$}

Errors in $\mathrm{S}_{21}$ from ferromagnetic resonance (FMR) measurements were calculated as the standard deviation of the measured spectra from the fitted spectra, as shown in Table S1. The FMR spectra is normalized between 0 and 1, the calculated error is also in the same range. Error usually decreases as microwave frequency increases. For example, as shown in Figure S7, which shows normalized $\mathrm{S}_{21}$ with errors at 16, 18, 20, and $22 \mathrm{GHz}$ for Fe MNWs with parallel orientation, there is a significant difference between two $\mathrm{S}_{21}$ at 16 and $22 \mathrm{GHz}$. It is because the absorption null at $16 \mathrm{GHz}$ is not defined by the Kittel equation. FMR for Fe MNWs with parallel magnetizations is defined by a null at zero DC field for $20 \mathrm{GHz}$, followed by nulls along the Kittel equation above $20 \mathrm{GHz}$. So, the null at $16 \mathrm{GHz}$ is not due to a well-behaved precessional resonance like FMR. One of the main points of this paper is to identify the FMR range for Fe, Co, and Ni MNWs that will yield the most efficient FMR identification, and these errors confirm the choice of using frequencies at $20 \mathrm{GHz}$ or above. 
Table S1. Errors in $\mathrm{S}_{21}$ from FMR measurements.

\begin{tabular}{|c|c|c|c|c|c|c|c|c|c|}
\hline \multicolumn{2}{|c|}{} & \multicolumn{8}{|c|}{ Microwave frequency [GHz] } \\
\cline { 2 - 10 } \multicolumn{1}{|c|}{} & 10 & 12 & 14 & 16 & 18 & 20 & 22 & 24 \\
\hline \multirow{4}{*}{ Error $( \pm)$} & $\mathrm{Fe} \|$ & 0.0549 & 0.0775 & 0.1008 & 0.0642 & 0.0088 & 0.0056 & 0.0035 & 0.0034 \\
\cline { 2 - 10 } & $\mathrm{Fe} \perp$ & 0.0393 & 0.0134 & 0.0075 & 0.0090 & 0.0058 & 0.0043 & 0.0043 & 0.0045 \\
\cline { 2 - 10 } & $\mathrm{Co} \|$ & 0.0604 & 0.0605 & 0.0592 & 0.0184 & 0.0048 & 0.0032 & 0.0024 & 0.0023 \\
\cline { 2 - 10 } & $\mathrm{Co} \perp$ & 0.0351 & 0.0126 & 0.0053 & 0.0047 & 0.0034 & 0.0030 & 0.0039 & 0.0041 \\
\cline { 2 - 10 } & $\mathrm{Ni} \|$ & 0.0368 & 0.0308 & 0.0184 & 0.0231 & 0.0370 & 0.0354 & 0.0363 & 0.0467 \\
\cline { 2 - 10 } & $\mathrm{Ni} \perp$ & 0.0299 & 0.0344 & 0.0355 & 0.0274 & 0.0437 & 0.0449 & 0.0562 & 0.0471 \\
\hline
\end{tabular}

\begin{tabular}{|c|c|c|c|c|c|c|c|c|c|}
\hline \multicolumn{2}{|c|}{} & \multicolumn{8}{c|}{ Microwave frequency [GHz] } \\
\cline { 2 - 10 } \multicolumn{1}{|c|}{} & 26 & 28 & 30 & 32 & 34 & 36 & 38 & 40 \\
\hline \multirow{4}{*}{ Error $( \pm)$} & $\mathrm{Fe} \|$ & 0.0035 & 0.0025 & 0.0030 & 0.0033 & 0.0038 & 0.0040 & 0.0036 & 0.0032 \\
\cline { 2 - 10 } & $\mathrm{Fe} \perp$ & 0.0055 & 0.0041 & - & - & - & - & - & - \\
\cline { 2 - 10 } & $\mathrm{Co} \|$ & 0.0025 & 0.0021 & 0.0023 & 0.0022 & 0.0018 & 0.0020 & 0.0019 & 0.0021 \\
\cline { 2 - 10 } & $\mathrm{Co} \perp$ & 0.0044 & 0.0039 & 0.0034 & 0.0027 & - & - & - & - \\
\cline { 2 - 10 } & $\mathrm{Ni} \|$ & 0.0150 & 0.0250 & 0.0262 & 0.0234 & 0.0403 & 0.0224 & 0.0202 & 0.0232 \\
\cline { 2 - 10 } & $\mathrm{Ni} \perp$ & 0.0170 & 0.0267 & 0.0275 & 0.0188 & 0.0468 & 0.0203 & 0.0164 & 0.0240 \\
\hline
\end{tabular}

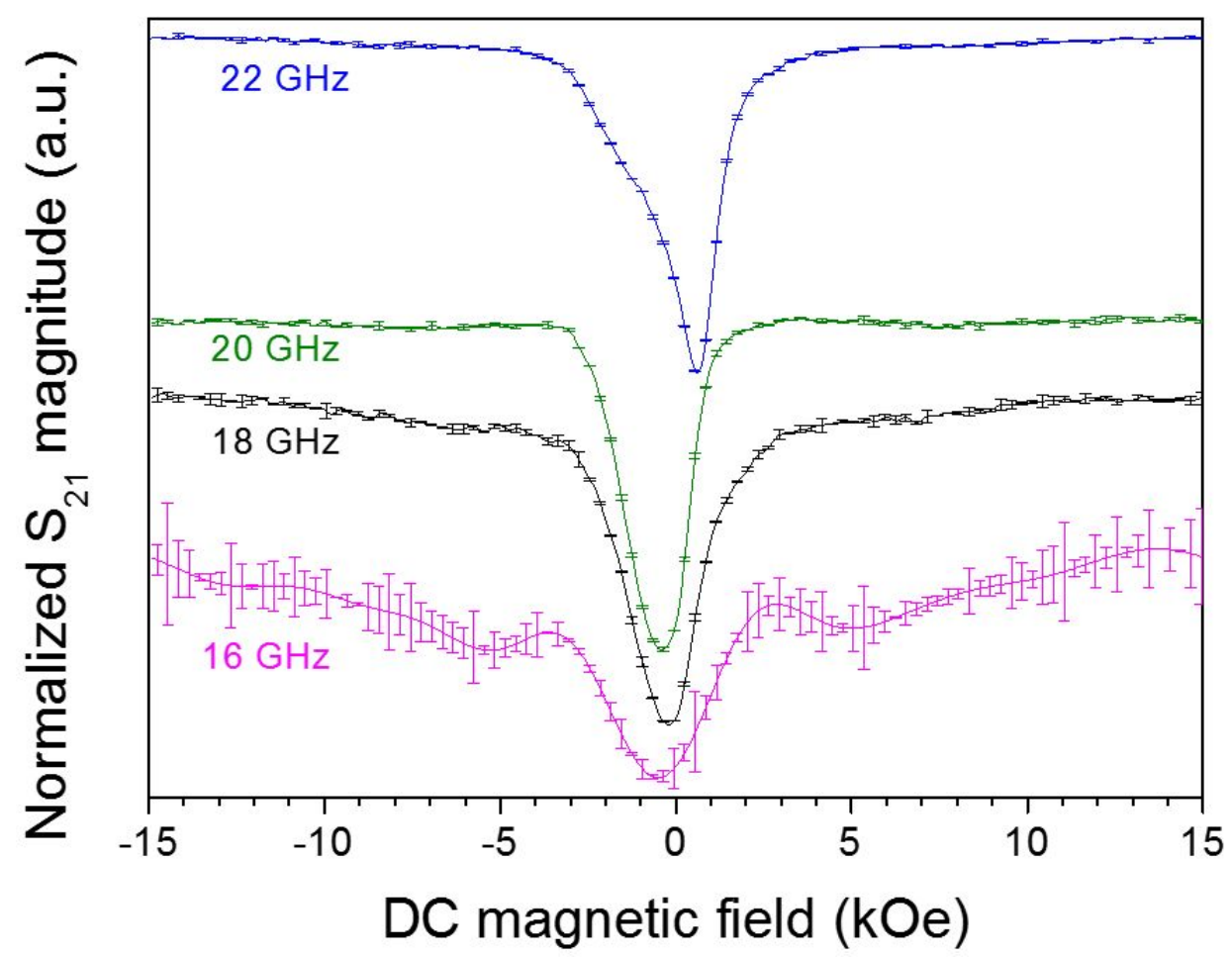

Figure S7. Stacked normalized $S_{21}$ (microwave signal at the output compared to the input) with errors at 16, 18, 20, and $22 \mathrm{GHz}$ for Fe MNWs with DC field parallel to MNW axis. 


\section{Errors in g-factor}

As shown in Figure 4, the experimental FMR trends of Fe, Co, and Ni MNWs with parallel magnetizations match the case 1 Kittel equation well with small errors due to small differences in slope. This slope difference comes from the difference in the g-factor of the gyromagnetic ratio. Table S2 shows the bulk g-factors from literature that was used in the Kittel equation and the experimental g-factors acquired by the line fitting of the FMR results at parallel orientation. The experimental g-factor depends on the local ordering or local magnetic fields, which are affected by the amount of defect, strain, and crystallinity inside the material. In this work, $\mathrm{Fe}, \mathrm{Co}$, and $\mathrm{Ni}$ MNWs grown in the confined nanopores of templates by electrodeposition were used for FMR measurements, and they have nanosized polycrystalline structures. It is reasonable that the experimental g-factors are slightly different from the bulk ones as found here.

Table S2. g-factors used in the Kittel equation and acquired by the line fitting of the results of FMR experiments with parallel orientation.

\begin{tabular}{|c|c|c|}
\hline \multirow{2}{*}{} & \multicolumn{2}{|c|}{ g-factor } \\
\cline { 2 - 3 } & Kittel equation & Experiment \\
\hline Fe & 2.08 & 1.874 \\
\hline $\mathrm{Co}$ & 2.145 & 1.986 \\
\hline $\mathrm{Ni}$ & 2.185 & 1.848 \\
\hline
\end{tabular}

